\title{
The rational homotopy type of the complement of the graph of a map
}

\author{
Angom Tiken Singh • Himadri Kumar Mukerjee
}

Received: 30 August 2011 / Accepted: 25 November 2014 / Published online: 16 December 2014

(C) Tbilisi Centre for Mathematical Sciences 2014

\begin{abstract}
We prove that the rational homotopy type of the complement of the graph of a continuous map from a simply connected closed manifold to a 2-connected closed manifold of the same dimension depends only on the rational homotopy class of the map. We give a commutative differential graded algebra model (in the sense of Sullivan) of the complement of the graph and study its formality.
\end{abstract}

Keywords Commutative differential graded algebra $\cdot$ Sullivan model · Minimal model · Formal space and map · Leray spectral sequence

Mathematics Subject Classification 55P62 - 55M05 - 55N30 - 55R20 - 55U25

\section{Introduction}

In this paper we have considered only smooth manifolds. However using the results of Milnor [14], Kister [8] and Kirby-Siebenmann [7] the arguments given for smooth manifolds can be carried over to topological manifolds.

Let $f: M \rightarrow N$ be a continuous map of closed connected oriented manifolds of dimension $n$. We aim to study the rational homotopy of $M \times N \backslash \Gamma(f)$, where $\Gamma(f)=\{(x, f(x)) \mid x \in M\}$ is the graph of $f$.

Communicated by Pascal Lambrechts.

A. T. Singh · H. K. Mukerjee $(\bowtie)$

Department of Mathematics, North-Eastern Hill University, NEHU Campus,

Shillong 793022, India

e-mail: himadrinehu@gmail.com

A. T. Singh

e-mail: angomtiken@gmail.com 
We refer the reader to [3] and [5] for standard definitions and terminologies of: Free commutative differential graded algebra (CDGA), (minimal) Sullivan CDGA, (minimal) Sullivan model for a CDGA and for a space X, homotopy between morphisms of CDGAs, formality of spaces.

Definition 1.1 ([5], [22], [4], [12], [20]) A model for a continuous map $f$ : $X \rightarrow Y$ between connected topological spaces is a morphism of CDGAs, $\psi_{f}$ : $\left(A_{Y}, d_{A_{Y}}\right) \rightarrow\left(A_{X}, d_{A_{X}}\right)$, such that there exists a homotopy commutative diagram, where $\left(\Lambda W, d_{W}\right),\left(\Lambda V, d_{V}\right)$ are minimal models of $Y$ and $X$ respectively and the vertical arrows are quasi-isomorphisms:

$$
\begin{array}{lll}
\left(A_{Y}, d_{A_{Y}}\right) & \stackrel{\psi_{f}}{\rightarrow} & \left(A_{X}, d_{A_{X}}\right) \\
\cong_{q} \uparrow \phi_{W} & & \cong_{q} \uparrow \phi_{V} \\
\left(\Lambda W, d_{W}\right) & \stackrel{\psi_{\Lambda f}}{\rightarrow} & \left(\Lambda V, d_{V}\right) \\
\cong_{q \downarrow} \downarrow \rho_{W} & & \cong_{q \downarrow} \downarrow \rho_{V} \\
\left(A_{P L}(Y), d_{Y}\right) \stackrel{A_{P L}(f)}{\rightarrow}\left(A_{P L}(X), d_{X}\right) .
\end{array}
$$

A continuous map $f: X \rightarrow Y$ between formal spaces is said to be formal (also referred to as formalizable in the literature $)$ if $f^{*}:\left(H^{*}(Y ; \mathbb{Q}), 0\right) \rightarrow\left(H^{*}(X ; \mathbb{Q}), 0\right)$ is a model for $f$.

Many authors have studied rational homotopy type of maps and their formality; see e.g. $[4,13,16-18,22]$.

These authors have studied spaces $C(f)$ closely associated with $f$, like the mapping cone of $f$, the cofibre of $f$, the homotopy fibre of $f$ and have recorded results related to problems of following types:

Problem 1.2 1. Find out if the rational homotopy of $C(f)$ is determined by the rational homotopy of $f$.

2. Given that $f$ is formal, determine if $C(f)$ is necessarily formal.

We study the following problems of a similar nature:

Problem 1.3 1. Find out if the rational homotopy of $M \times N \backslash \Gamma(f)$, where $\Gamma(f)$ is the graph of $f$, is determined by the rational homotopy of $f$.

2. Given that $f$ is formal, determine if $M \times N \backslash \Gamma(f)$ is necessarily formal.

The motivation of this paper comes from the work of Lambrechts and Stanley [9] on the rational homotopy type of configuration spaces of two points, and the observation that if $f$ is the identity map $1_{M}: M \rightarrow M$ then, the graph of $f, \Gamma(f)=\Delta$, the diagonal, and $M \times M \backslash \Gamma(f)=M \times M \backslash \Delta=F(M, 2)$, the configuration space of two points. We begin with the following immediate observations:

1. If $f: \mathbb{R}^{n} \rightarrow \mathbb{R}^{n}$ is any continuous map then $\mathbb{R}^{n} \times \mathbb{R}^{n} \backslash \Gamma(f)$ is homeomorphic to $F\left(\mathbb{R}^{n}, 2\right)$ under the homeomorphism $(u, v) \mapsto(u, v-f(u)+u)$. 
2. For a topological space $X$ if $f: X \rightarrow X$ is a homeomorphism then $X \times X \backslash \Gamma(f)$ is homeomorphic to $X \times X \backslash \Delta=F(X, 2)$ under the homeomorphism $(x, y) \mapsto$ $\left(x, f^{-1}(y)\right)$.

3. Suppose that $X=S^{2} \vee S^{3}, f: X \rightarrow X$ is the constant map $x \mapsto(0,0,1)$ and $g: X \rightarrow X$ is the constant map $x \mapsto(0,0,0,-1)$. Then $X \times X \backslash \Gamma(f) \simeq$ $X \times\left(\mathbb{R}^{2} \vee S^{3}\right)$ whereas $X \times X \backslash \Gamma(g) \simeq X \times\left(S^{2} \vee \mathbb{R}^{3}\right)$. Therefore they are not homotopy equivalent. Thus for an arbitrary space $X$ and a continuous map $f: X \rightarrow X, X \times X \backslash \Gamma(f)$ need not even be homotopy equivalent to $F(X, 2)$.

Lambrechts and Stanley $[9,10]$ proved that if $M$ is a simply-connected closed manifold such that $H^{2}(M ; \mathbb{Q})=0$ then the rational homotopy type of $F(M, 2)$ depends only on the rational homotopy type of $M$. They also proved in [9] that if $M$ is a 2-connected closed manifold, then $F(M, 2)$ is formal if and only if $M$ is formal by constructing explicitly a CDGA model for $F(M, 2)$ out of a differential Poincaré duality algebra model of $M$ and a model of the diagonal map.

We show that starting from a CDGA model of $f: M \rightarrow N$ one can construct a CDGA model of $\left(1_{M} \times f\right) \circ \Delta_{M}: M \rightarrow M \times N$, where $\Delta_{M}(x)=(x, x)$ for $x \in M$. This allows us by Corollary 1.5 of [10], to conclude that if we take $M, N$ to be simply connected and $H^{2}(N ; \mathbb{Q})=0$ then the rational homotopy type of $M \times N \backslash \Gamma(f)$ is determined by the rational homotopy class of $f$ :

Theorem 1.4 Let $f: M \rightarrow N$ be a continuous map of closed connected oriented manifolds of dimension $n$ such that $H^{1}(M ; \mathbb{Q})=0=H^{1}(N ; \mathbb{Q})=H^{2}(N ; \mathbb{Q})$, then a CDGA-model of $M \times N \backslash \Gamma(f)$ can be explicitly determined out of any CDGA-model of $f$.

Corollary 1.5 If $f: M \rightarrow N$ is a continuous map of closed simply connected manifolds of dimension $n$ such that $H^{2}(N ; \mathbb{Q})=0$, then the rational homotopy type of $M \times N \backslash \Gamma(f)$ depends only on the rational homotopy class of $f$.

We relativize results of [9] by defining a class $\Delta_{\psi}$ depending on a morphism $\psi$ of differential Poincaré duality algebras similar to the diagonal class $\Delta$ in [9]. We know from Theorem 1.1 of [11] that if $M, N$ are closed connected oriented manifolds with $H^{1}(M ; \mathbb{Q})=0=H^{1}(N ; \mathbb{Q})$, then they admit differential Poincaré duality algebra models. We also know from Proposition 1 of [2] that if $f: M \rightarrow N$ is a continuous map of simply connected manifolds of dimension $n \geq 7$ (it is a conjecture that this dimensional restriction can be removed) such that $H^{2}(f)$ is injective, then $f$ admits a Sullivan model $\psi_{f}:\left(A_{N}, d_{A_{N}}\right) \rightarrow\left(A_{M}, d_{A_{M}}\right)$, where $\left(A_{M}, d_{A_{M}}\right),\left(A_{N}, d_{A_{N}}\right)$ are Poincaré duality algebras. Thus assuming the existence of such a model of $f$ we can construct a specific CDGA model of $M \times N \backslash \Gamma(f)$ from the model of $f$ :

Theorem 1.6 Let $f: M \rightarrow N$ be a continuous map of closed connected oriented manifolds of dimension $n$ such that $H^{1}(M ; \mathbb{Q})=0=H^{1}(N ; \mathbb{Q})=H^{2}(N ; \mathbb{Q})$. If $\psi_{f}:\left(A_{N}, d_{A_{N}}\right) \rightarrow\left(A_{M}, d_{A_{M}}\right)$ is a model of $f$, where $\left(A_{M}, d_{A_{M}}\right),\left(A_{N}, d_{A_{N}}\right)$ are oriented differential Poincaré duality algebras, $\left\{a_{i}\right\}_{1 \leq i \leq l}$ a homogeneous basis of $A_{N}$ and $\left\{a_{i}^{*}\right\}_{1 \leq i \leq l}$ its Poincaré dual basis 


$$
\Delta=\sum_{i=1}^{l_{N}}(-1)^{\operatorname{deg}\left(a_{i}\right)} a_{i} \otimes a_{i}^{*} \in\left(A_{N} \otimes A_{N}\right)^{n}
$$

is the diagonal class (defined in [9], $p .1030)$ and $\Delta_{\psi_{f}}:=\left(\psi_{f} \otimes 1\right)(\Delta)$, called the class of $\psi_{f}$, then the ideal $\left(\Delta_{\psi_{f}}\right)=\Delta_{\psi_{f}} .\left(A_{M} \otimes A_{N}\right)$ is a differential ideal of $A_{M} \otimes A_{N}$, and the quotient $C D G A$

$$
\left(\frac{A_{M} \otimes A_{N}}{\left(\Delta_{\psi_{f}}\right)}, \overline{d_{A_{M}} \otimes d_{A_{N}}}\right)
$$

is a CDGA model of $M \times N \backslash \Gamma(f)$.

This allows us to prove that if $f$ is formal then $M \times N \backslash \Gamma(f)$ is also formal.

Corollary 1.7 Let $f: M \rightarrow N$ be a formal map of closed connected oriented formal manifolds of dimension $n$ such that $H^{1}(M ; \mathbb{Q})=0=H^{1}(N ; \mathbb{Q})=H^{2}(N ; \mathbb{Q})$, then $M \times N \backslash \Gamma(f)$ is a formal space.

As the first major step in this paper we compute the cohomology algebra $H^{*}(M \times$ $N \backslash \Gamma(f))$ using the Leray spectral sequence as described in $\$ 2$ of Totaro [21]. We analyze at depth the cohomology class $\mu_{f}$, as described in Chapter 30 of Greenberg and Harper [6].

Let $\tilde{\mu} \in H^{n}(M \times N, M \times N \backslash \Gamma(f) ; \mathbb{Z})$ be the Thom class as in Lemma 2.1, and $\tilde{\mu^{\prime}}=j^{*}(\tilde{\mu}) \in H^{n}(M \times N ; \mathbb{Z})$, where $j: M \times N \rightarrow(M \times N, M \times N \backslash \Gamma(f))$ is the natural injection. Let us now take cohomology with rational coefficients (or coefficients in any field). Let $\left\{b_{i}\right\}_{1 \leq i \leq l_{N}}$ be a homogeneous basis of $H^{*}(N ; \mathbb{Q})$, and let $\left\{b_{i}^{*}\right\}_{1 \leq i \leq l_{N}}$ be its Poincaré dual basis, that is $\left\langle b_{i} \cup b_{j}^{*},[N]\right\rangle=\delta_{i j}$, where $[N]$

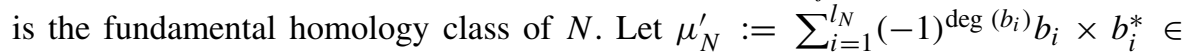
$\left(H^{*}(N \times N ; \mathbb{Q})\right)^{n}$ be the diagonal class of $N$, and let $\mu_{f}:=\left(f \times i d_{N}\right)^{*}\left(\mu_{N}^{\prime}\right)$, called the cohomology class of the graph of $f$ (see p. 284 of [6]). Then there is a unique class $\Delta_{f} \in\left(H^{*}(M ; \mathbb{Q}) \otimes H^{*}(N ; \mathbb{Q})\right)^{n}$ which maps to $\mu_{f}$ under the isomorphism of the Künneth theorem $\left(H^{*}(M ; \mathbb{Q}) \otimes H^{*}(N ; \mathbb{Q})\right)^{n} \stackrel{\cong}{\rightarrow}\left(H^{*}(M \times N ; \mathbb{Q})\right)^{n}$, we call $\Delta_{f}$ the class of $f$.

Theorem 1.8 Let $f: M \rightarrow N$ be a continuous map of closed oriented manifolds of dimension $n \geq 2$ and $\Gamma(f)$ be the graph of $f$. Then we have the following isomorphism of rings:

$$
H^{*}(M \times N \backslash \Gamma(f) ; \mathbb{Z}) \cong \frac{H^{*}(M \times N ; \mathbb{Z})}{\left(\tilde{\mu}^{\prime}\right)},
$$

where $\left(\tilde{\mu}^{\prime}\right)$ denotes the ideal $H^{*}(M \times N ; \mathbb{Z}) \cup \tilde{\mu}^{\prime}$. For coefficients in $\mathbb{Q}$, and $\Delta_{f}=$ $\sum_{i=1}^{l_{N}}(-1)^{\operatorname{deg}\left(b_{i}\right)} f^{*}\left(b_{i}\right) \otimes b_{i}^{*}$, we have the following isomorphism of algebras:

$$
H^{*}(M \times N \backslash \Gamma(f) ; \mathbb{Q}) \cong \frac{H^{*}(M \times N ; \mathbb{Q})}{\left(\mu_{f}\right)} \cong \frac{H^{*}(M ; \mathbb{Q}) \otimes H^{*}(N ; \mathbb{Q})}{\left(\Delta_{f}\right)} .
$$


The paper is arranged as follows. In $\S 2$ we determine the cohomology algebra of $M \times N \backslash \Gamma(f)$. In $\S 3$ we adapt for a continuous map $f: M \rightarrow N$ some results of [9] and [10] to construct a CDGA model of $M \times N \backslash \Gamma(f)$, and prove Theorem 1.4 and Corollary 1.5. In $\S 4$ we prove several results about differential Poincaré duality algebra associated to a morphism $\psi$ by adapting similar results of [9], proved in the absolute case. These results and some results of [10] are used for the proofs of Theorem 1.6 and Corollary 1.7. In the final $\S 5$ we give some examples and applications of our results.

\section{The cohomology algebra of $M \times N \backslash \Gamma(f)$}

In this section we prove Theorem 1.8.

We assume that $f: M \rightarrow N$ is a continuous map of closed connected oriented manifolds of dimension $n$ with graph $\Gamma(f)$. Since $\Gamma(f) \subset M \times N$ is an embedding, one can derive the following analogue of Corollary 11.2 of Milnor-Stasheff [15] (and also of Corollary (30.2) of Greenberg-Harper [6]) in a similar fashion. We follow the notations which preceded the statement of Theorem 1.8 in $\S 1$.

Lemma 2.1 There is a Thom class $\tilde{\mu} \in H^{n}(M \times N, M \times N \backslash \Gamma(f)$; $\mathbb{Z})$ associated to the oriented normal bundle of the embedding $\Gamma(f) \subset M \times N$.

We next prove the following analogue of Corollary (30.3) of [6]:

Theorem 2.2 Let $[N] \in H_{n}(N ; \mathbb{Z})$ be the fundamental homology class of $N$, $j^{*}: H^{n}(M \times N, M \times N \backslash \Gamma(f) ; \mathbb{Z}) \rightarrow H^{n}(M \times N ; \mathbb{Z})$ be the homomorphism in cohomology induced by the map $j: M \times N \hookrightarrow(M \times N, M \times N \backslash \Gamma(f))$ and $\tilde{\mu}^{\prime}=j^{*}(\tilde{\mu})$, then $\tilde{\mu}^{\prime} /[N]=1$.

Proof For $x \in M$, consider the commutative diagram:

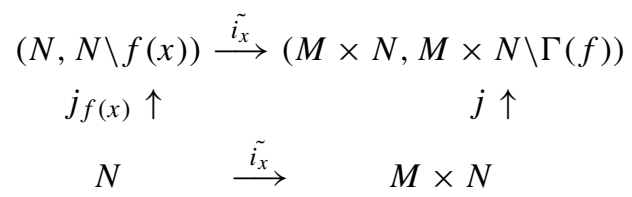

where $\tilde{i_{x}}:(N, N \backslash f(x)) \rightarrow(M \times N, M \times N \backslash \Gamma(f))$ is defined by $\tilde{i_{x}}\left(x^{\prime}\right)=$ $\left(x, x^{\prime}\right), \forall x^{\prime} \in N, i_{x}: N \rightarrow M \times N$ its restriction to $(N, \emptyset)$ and $j_{f(x)}: N \rightarrow$ $(N, N \backslash f(x))$ is the natural injection. If $s: N \rightarrow N^{0}$ is a section of the orientation sheaf $N^{0}$ over $N$, then

$$
\begin{aligned}
1 & =\left[s(f(x)),{\tilde{i_{x}}}^{*}(\tilde{\mu})\right]=\left[j_{f(x)_{*}}[N],{\tilde{i_{x}}}^{*}(\tilde{\mu})\right]=\left[[N], j_{f(x)}^{*} \tilde{i_{x}^{*}}(\tilde{\mu})\right] \\
& =\left[[N],\left(\tilde{i_{x}} \circ j_{f(x)}\right)^{*}(\tilde{\mu})\right]=\left[[N],\left(j \circ \tilde{i_{x}}\right)^{*}(\tilde{\mu})\right]=\left[[N], \tilde{i_{x}}{ }^{*} \circ j^{*}(\tilde{\mu})\right] \\
& =\left[\tilde{i_{x *}}[N], j^{*}(\tilde{\mu})\right]=\left[\tilde{i_{x *}}[N], \tilde{\mu}^{\prime}\right] .
\end{aligned}
$$

Since $N \cong\{x\} \times N$, if $\bar{x}$ is the homology class of the zero cycle $x, \tilde{i_{x *}}([N])=$ $\bar{x} \times[N]$. So from the above expression we get 
$1=\left[\tilde{i_{x *}}[N], \tilde{\mu}^{\prime}\right]=\left[\bar{x} \times[N], \tilde{\mu}^{\prime}\right]=\left[\bar{x}, \tilde{\mu}^{\prime} /[N]\right]$ for all $x \in M$, which proves the result.

We now prove the following analogue of Lemma 11.8 of [15] (and also of Lemma (30.5) of [6]):

Theorem 2.3 If $a \in H^{*}(N ; \mathbb{Z})$, then $\left(f^{*}(a) \times 1\right) \cup \tilde{\mu}^{\prime}=(1 \times a) \cup \tilde{\mu}^{\prime}$.

Proof Let $V_{\Gamma(f)}$ be a tubular neighborhood of the graph $\Gamma(f)$ in $M \times N$. So $\Gamma(f)$ is a deformation retract of $V_{\Gamma(f)}$. Let $i_{\Gamma(f)}: \Gamma(f) \rightarrow V_{\Gamma(f)}$ be the inclusion map and $r_{\Gamma(f)}: V_{\Gamma(f)} \rightarrow \Gamma(f)$ be the retraction map. Then $r_{\Gamma(f)} \circ i_{\Gamma(f)}=1_{\Gamma(f)}$ and $i_{\Gamma(f)} \circ r_{\Gamma(f)} \simeq 1_{V_{\Gamma(f)}}$. Consider the projections $p_{1}: M \times N \rightarrow M$ and $p_{2}: M \times N \rightarrow$ $N$. Since $f \circ p_{1}$ and $p_{2}$ coincide on $\Gamma(f)$, we have $\left.\left(f \circ p_{1}\right)\right|_{V_{\Gamma(f)}} \circ i_{\Gamma(f)} \circ r_{\Gamma(f)}=$ $\left.p_{2}\right|_{V_{\Gamma(f)}} \circ i_{\Gamma(f)} \circ r_{\Gamma(f)}$. So the $\left.\left.\left(f \circ p_{1}\right)\right|_{V_{\Gamma(f)}} \simeq p_{2}\right|_{V_{\Gamma(f)}}$. Therefore the cohomology classes $\left(f \circ p_{1}\right)^{*}(a)=p_{1}^{*} \circ f^{*}(a)=f^{*}(a) \times 1$ and $p_{2}^{*}(a)=1 \times a$ have the same image under the restriction homomorphism $H^{i}(M \times N ; \mathbb{Z}) \rightarrow H^{i}\left(V_{\Gamma(f)} ; \mathbb{Z}\right)$. Now using the commutative diagram

$$
\begin{array}{ccc}
H^{i}(M \times N ; \mathbb{Z}) & H^{i}\left(V_{\Gamma(f)} ; \mathbb{Z}\right) \\
\downarrow \cup \tilde{\mu} & \left.\downarrow \cup \tilde{\mu}\right|_{\left(V_{\Gamma(f)}, V_{\Gamma(f)} \backslash \Gamma(f)\right)} \\
H^{i+n}(M \times N, M \times N \backslash \Gamma(f) ; \mathbb{Z}) \cong H^{i+n}\left(V_{\Gamma(f)}, V_{\Gamma(f)} \backslash \Gamma(f) ; \mathbb{Z}\right)
\end{array}
$$

it follows that $\left(f^{*}(a) \times 1\right) \cup \tilde{\mu}=(1 \times a) \cup \tilde{\mu}$. So $j^{*}\left(\left(f^{*}(a) \times 1\right) \cup \tilde{\mu}\right)=j^{*}((1 \times a) \cup$ $\tilde{\mu}), j$ as defined earlier. By the properties of mixed cup products $\left(f^{*}(a) \times 1\right) \cup j^{*}(\tilde{\mu})=$ $(1 \times a) \cup j^{*}(\tilde{\mu})$. So $\left(f^{*}(a) \times 1\right) \cup \tilde{\mu}^{\prime}=(1 \times a) \cup \tilde{\mu}^{\prime}$.

The next theorem is an analogue of Theorem 11.11 of [15] (and also of Proposition (30.18) of [6]):

Theorem 2.4 Let $\left\{b_{i}\right\}_{i=1}^{l_{N}}$ be a homogeneous basis of $H^{*}(N ; \mathbb{Q})$, and let $\left\{b_{i}^{*}\right\}_{i=1}^{l_{N}}$ be its Poincaré dual basis, then the cohomology class $\tilde{\mu}^{\prime}$ is given by

$$
\tilde{\mu}^{\prime}=\sum_{i=1}^{l_{N}}(-1)^{\operatorname{deg}\left(b_{i}\right)} f^{*}\left(b_{i}\right) \times b_{i}^{*},
$$

where deg $\left(b_{i}\right)=k$ if $b_{i} \in H^{k}(N ; \mathbb{Q})$.

Proof Using the Künneth formula we can write $\tilde{\mu}^{\prime} \in H^{n}(M \times N ; \mathbb{Q})$ as

$$
\tilde{\mu}^{\prime}=c_{1} \times b_{1}^{*}+\ldots+c_{l_{N}} \times b_{l_{N}}^{*},
$$

where $c_{1}, \ldots, c_{l_{N}}$ are certain well defined cohomology classes in $H^{*}(M ; \mathbb{Q})$ with $\operatorname{deg}\left(b_{i}\right)+\operatorname{deg}\left(c_{i}\right)=n$. We apply the homomorphism $/[N]$ on both sides of the identity $\left(f^{*}(a) \times 1\right) \cup \tilde{\mu}^{\prime}=(1 \times a) \cup \tilde{\mu}^{\prime}$, where $a \in H^{*}(N ; \mathbb{Q})$. By Property 4 of slant product on p. 288 of Spanier [19] and by our Theorem 2.2 we get

$$
\left(\left(f^{*}(a) \times 1\right) \cup \tilde{\mu}^{\prime}\right) /[N]=f^{*}(a) \cup\left(\tilde{\mu}^{\prime} /[N]\right)=f^{*}(a) .
$$


Also,

$$
\begin{aligned}
\left((1 \times a) \cup \tilde{\mu}^{\prime}\right) /[N]= & (1 \times a) \cup \sum_{i=1}^{l_{N}} c_{i} \times b_{i}^{*} /[N]=\sum_{i=1}^{l_{N}}(-1)^{\operatorname{deg}(a) \operatorname{deg}\left(c_{i}\right)} c_{i} \\
& \times\left(a \cup b_{i}^{*}\right) /[N] \\
= & \sum_{i=1}^{l_{N}}(-1)^{\operatorname{deg}(a) \operatorname{deg}\left(c_{i}\right)} c_{i}\left\langle a \cup b_{i}^{*},[N]\right\rangle
\end{aligned}
$$

Using equations (1) and (2) above and substituting $b_{j}$ for $a$, we get

$$
f^{*}\left(b_{j}\right)=\sum_{i=1}^{l_{N}}(-1)^{\operatorname{deg}\left(b_{j}\right) \operatorname{deg}\left(c_{i}\right)} c_{i}\left\langle b_{j} \cup b_{i}^{*},[N]\right\rangle=(-1)^{\operatorname{deg}\left(b_{j}\right) \operatorname{deg}\left(c_{j}\right)} c_{j},
$$

but $\operatorname{deg}\left(b_{j}\right)=\operatorname{deg}\left(c_{j}\right)$, so we get that

$(-1)^{\operatorname{deg}\left(b_{j}\right)} f^{*}\left(b_{j}\right)=(-1)^{\operatorname{deg}\left(b_{j}\right)}(-1)^{\operatorname{deg}\left(b_{j}\right) \operatorname{deg}\left(b_{j}\right)} c_{j}=(-1)^{\operatorname{deg}\left(b_{j}\right)\left(1+\operatorname{deg}\left(b_{j}\right)\right.} c_{j}=c_{j}$.

This proves the theorem.

Let $[\Gamma(f)]=\left(1_{M} \times f\right)_{*} \circ \Delta_{M *}([M])$. Let $\mu_{N} \in H^{n}\left(N \times N, N \times N \backslash \Gamma\left(1_{N}\right) ; \mathbb{Z}\right)$ be the Thom class of the oriented normal bundle of the embedding $\Gamma\left(1_{N}\right) \hookrightarrow N \times N$, let $\mu_{N}^{\prime} \in H^{n}(N \times N ; \mathbb{Z})$ be its image under the homomorphism in cohomology induced by $j_{N}: N \times N \rightarrow\left(N \times N, N \times N \backslash \Gamma\left(1_{N}\right)\right)$ and $\mu_{f}=\left(f \times 1_{N}\right)^{*}\left(\mu_{N}^{\prime}\right) \in$ $H^{n}(M \times N ; \mathbb{Z})$.

Remark 2.5 1. When coefficients belong to $\mathbb{Q}$ (or any field) by Theorem 11.11 of [15] (or by Proposition (30.18) of [6]), if $\left\{a_{i}\right\}_{i=1}^{l_{M}}$ is a homogeneous basis of $H^{*}(M ; \mathbb{Q})$ and $\left\{a_{i}^{*}\right\}_{i=1}^{l_{M}}$ its Poincaré dual basis, we have $\mu_{M}^{\prime}=\sum_{i=1}^{l_{M}}(-1)^{\operatorname{deg}\left(a_{i}\right)} a_{i} \times a_{i}^{*}$, and $\mu_{N}^{\prime}=\sum_{i=1}^{l_{N}}(-1)^{\operatorname{deg}\left(b_{i}\right)} b_{i} \times b_{i}^{*}$. Therefore, we see using Theorem 2.4 , that in rational cohomology, $\mu_{f}=\tilde{\mu}^{\prime}$.

2. By Exercises (30.15) and (30.17) of [6] we have $[M \times M] \cap \mu_{M}^{\prime}=\Delta_{M *}([M])=$ $\left[\Gamma\left(1_{M}\right)\right]$, and $[N \times N] \cap \mu_{N}^{\prime}=\Delta_{N *}([N])=\left[\Gamma\left(1_{N}\right)\right]$. Using this and Part 1 of this remark one can prove the following statement which is the assertion of the Exercise (30.22) of [6]:

$$
[M] \times[N] \cap \tilde{\mu}^{\prime}=[M] \times[N] \cap \mu_{f}=[\Gamma(f)]
$$

We are now ready to prove Theorem 1.8.

Proof of Theorem.1.8 Let $h: M \times N \backslash \Gamma(f) \hookrightarrow M \times N$ denote the inclusion map. The Leray spectral sequence for this inclusion has the form

$$
E_{2}^{i, j}=H^{i}\left(M \times N ; R^{j} h_{*} \mathbb{Z}\right) \Rightarrow H^{i+j}(M \times N \backslash \Gamma(f) ; \mathbb{Z}) .
$$


Here $R^{j} h_{*} \mathbb{Z}$ is the Leray sheaf on $M \times N$ associated with the presheaf $U \rightarrow H^{j}(U \cap$ $(M \times N \backslash \Gamma(f)) ; \mathbb{Z})$ where $U$ runs over the family of all open subsets of $M \times N$. The stalk of $R^{j} h_{*} \mathbb{Z}$ at a point $\bar{x}=\left(x_{1}, x_{2}\right) \in M \times N$ is described below.

1. If $x_{2} \neq f\left(x_{1}\right)$, we can choose small coordinate neighborhoods $U_{1}$ and $U_{2}$ of $x_{1}$ and $x_{2}$ in $M$ and $N$ respectively, such that $f\left(U_{1}\right) \cap U_{2}=\emptyset$. If $U=U_{1} \times U_{2}$, then $U \subset M \times N \backslash \Gamma(f)$. Therefore, for every $\bar{x}=\left(x_{1}, x_{2}\right) \in M \times N, x_{2} \neq f\left(x_{1}\right)$, $\left(R^{j} h_{*} \mathbb{Z}\right)_{\bar{x}}=H^{j}(U \cap(M \times N \backslash \Gamma(f)) ; \mathbb{Z})=H^{j}(U ; \mathbb{Z})=H^{j}\left(U_{1} \times U_{2} ; \mathbb{Z}\right)=0$ if $j \neq 0$, and $\left(R^{0} h_{*} \mathbb{Z}\right)_{\bar{x}}=\mathbb{Z}$.

2. If $x_{2}=f\left(x_{1}\right)$, we can choose small coordinate neighborhoods $U_{1}$ and $U_{2}$ of $x_{1}$ and $x_{2}$ in $M$ and $N$ respectively, such that $f\left(U_{1}\right) \subset U_{2}$. For $U=U_{1} \times U_{2}$, $\left(R^{j} g_{*} \mathbb{Z}\right)_{\bar{x}}=H^{j}(U \cap(M \times N \backslash \Gamma(f)) ; \mathbb{Z})=H^{j}(U \backslash \Gamma(f) ; \mathbb{Z})=H^{j}\left(U_{1} \times U_{2} \backslash\right.$ $\Gamma(f) ; \mathbb{Z})$. Let $h_{1}: U_{1} \stackrel{\cong}{\rightarrow} \mathbb{R}^{n}$ and $h_{2}: U_{2} \stackrel{\cong}{\rightarrow} \mathbb{R}^{n}$, be the coordinate charts of $U_{1}$ and $U_{2}$ respectively. Consider the composite $\hat{f}=h_{2} \circ f \circ h_{1}^{-1}: \mathbb{R}^{n} \rightarrow \mathbb{R}^{n}$, then $\Gamma(\hat{f})=h_{1} \times h_{2}\left(\left.\Gamma(f)\right|_{U_{1} \times U_{2}}\right)$. Therefore, $\left(R^{j} h_{*} \mathbb{Z}\right)_{\bar{x}}=H^{j}\left(U_{1} \times U_{2} \backslash \Gamma(f) ; \mathbb{Z}\right) \cong$ $H^{j}\left(\left(\mathbb{R}^{n} \times \mathbb{R}^{n}\right) \backslash \Gamma(\hat{f}) ; \mathbb{Z}\right)$. But as observed in the introduction $\mathbb{R}^{n} \times \mathbb{R}^{n} \backslash \Gamma(\hat{f})$ is homeomorphic to $F\left(\mathbb{R}^{n}, 2\right)$, so for every $\bar{x}=\left(x_{1}, x_{2}\right) \in M \times N, x_{2}=f\left(x_{1}\right)$

$$
\left(R^{j} h_{*} \mathbb{Z}\right)_{\bar{x}}=H^{j}\left(F\left(\mathbb{R}^{n}, 2\right) ; \mathbb{Z}\right)= \begin{cases}\mathbb{Z} & \text { if } j=0, n-1 \\ 0 & \text { otherwise }\end{cases}
$$

This shows that the Leray sheaf $\left(R^{n-1} h_{*} \mathbb{Z}\right)$ is supported and is locally constant along the graph $\Gamma(f)$ with stalks $\mathbb{Z}$, and $\left(R^{0} h_{*} \mathbb{Z}\right)$ is locally constant on $M \times N$ with stalks $\mathbb{Z}$. Since $M, N$ and hence $\Gamma(f)$ are orientable, we have

$$
H^{i}\left(M \times N ; R^{j} h_{*} \mathbb{Z}\right)= \begin{cases}H^{i}(M \times N ; \mathbb{Z}) & \text { if } j=0 \\ H^{i}(\Gamma(f) ; \mathbb{Z}) & \text { if } j=n-1, \\ 0 & \text { otherwise }\end{cases}
$$

Hence, the $E_{2}$ terms of Leray spectral sequence take the form:

$$
\begin{aligned}
& \begin{array}{ccccc}
0 & 0 & \cdots & 0 & \cdots \\
E_{2}^{0, n-1}= & H^{0}(\Gamma(f) ; \mathbb{Z}) E_{2}^{1, n-1}=H^{1}(\Gamma(f) ; \mathbb{Z}) & \cdots & E_{2}^{n, n-1}=H^{n}(\Gamma(f) ; \mathbb{Z}) \cdots \\
0 & 0 & \cdots & 0 & \cdots \\
0 & 0 & \cdots & 0 & \cdots \\
0 & 0 & \cdots & 0 & \cdots \\
0 & 0 & \cdots & 0 & \cdots
\end{array} \\
& E_{2}^{0,0}=H^{0}(M \times N ; \mathbb{Z}) E_{2}^{1,0}=H^{1}(M \times N ; \mathbb{Z}) \cdots E_{2}^{n, 0}=H^{n}(M \times N ; \mathbb{Z}) \cdots
\end{aligned}
$$

and $E_{n}$ terms coincide with $E_{2}$ terms: 


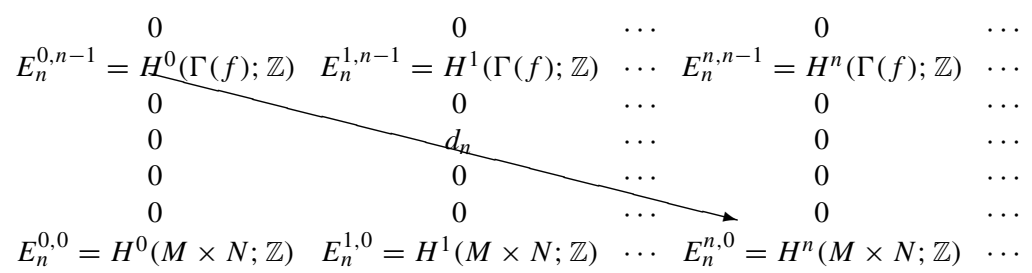

We now determine $d_{n}$ on the $E_{n}$ page. Note that the differential $d_{n}$ is 0 on the bottom row, since it maps each row to a lower row. Let $i: \Gamma(f) \rightarrow M \times N$ be the inclusion map and let $\pi: M \times N \rightarrow \Gamma(f)$ be defined by $\pi\left(x_{1}, x_{2}\right)=\left(x_{1}, f\left(x_{1}\right)\right)$. Then $\pi \circ i=1_{\Gamma(f)}$. Therefore, by the functoriality of cohomology, the homomorphisms induced in cohomology, $\pi^{*}$ and $i^{*}$ are, respectively, injective and surjective. Hence the long cohomology exact sequence of the pair $(M \times N, \Gamma(f))$ gives rise to the following split short exact sequence:

$$
0 \rightarrow H^{*}(M \times N, \Gamma(f) ; \mathbb{Z}) \stackrel{j_{1}^{*}}{\rightarrow} H^{*}(M \times N ; \mathbb{Z}) \underset{\pi^{*}}{\stackrel{i^{*}}{\rightleftharpoons}}\left(H^{*}(\Gamma(f) ; \mathbb{Z})\right) \rightarrow 0,
$$

where $j_{1}: M \times N \rightarrow(M \times N, \Gamma(f))$ is the natural injection.

As $\Gamma(f)$ is a smooth submanifold with an orientable normal bundle in the smooth manifold $M \times N$, the differentials $d_{n}$ in the above Leray spectral sequence originating from the $(n-1)^{t h}$ row are Gysin maps. If $z \in H^{i}(M \times N ; \mathbb{Z}), i^{*}(z) \in H^{i}(\Gamma(f) ; \mathbb{Z})$ and we have $d_{n}\left(i^{*}(z)\right)=\pi^{*}\left(i^{*}(z)\right) \cup \tilde{\mu}^{\prime}$. From the above split exact sequence we see that $z-\pi^{*}\left(i^{*}(z)\right)=j_{1}^{*}(w)$, for some element $w \in H^{i}(M \times N, \Gamma(f) ; \mathbb{Z})$. Recall that $\tilde{\mu}^{\prime}=j^{*}(\tilde{\mu})$, the image of the Thom class $\tilde{\mu} \in H^{*}(M \times N, M \times N \backslash \Gamma(f) ; \mathbb{Z})$, where $j: M \times N \rightarrow(M \times N, M \times N \backslash \Gamma(f))$ is the natural injection. So by Property 8 on p. 251 of [19] and the fact that $w \cup \tilde{\mu}=0$ (since the mixed cup product $\cup$ : $H^{i}(M \times N, \Gamma(f) ; \mathbb{Z}) \otimes H^{n}(M \times N, M \times N \backslash \Gamma(f) ; \mathbb{Z}) \rightarrow H^{i+n}(M \times N, \Gamma(f) \cup$ $M \times N \backslash \Gamma(f) ; \mathbb{Z})=H^{i+n}(M \times N, M \times N ; \mathbb{Z})=0$ has its image in a zero module) we get that $\left(z-\pi^{*}\left(i^{*}(z)\right)\right) \cup \tilde{\mu}^{\prime}=j_{1}^{*}(w) \cup \tilde{\mu}^{\prime}=j_{1}^{*}(w) \cup j^{*}(\tilde{\mu})=0$. This means, in particular, that $d_{n}(1)=\tilde{\mu}^{\prime}$.

If $k: M \rightarrow \Gamma(f)$ denotes the mapping $x \mapsto(x, f(x))$ and $\pi_{M}: M \times N \rightarrow M$ be the projection onto $M$, then $k^{*}$ is an isomorphism and $\pi^{*}=\pi_{M}^{*} \circ k^{*}$. Thus $d_{n}\left(i^{*}(z)\right)=\pi_{M}^{*}\left(k^{*}\left(i^{*}(z)\right)\right) \cup \tilde{\mu}^{\prime}=\left(k^{*}\left(i^{*}(z)\right) \times 1\right) \cup \tilde{\mu}^{\prime}$. But by Property 4 of slant products on p. 288 of [19], $\left\{\left(k^{*}\left(i^{*}(z)\right) \times 1\right) \cup \tilde{\mu}^{\prime}\right\} /[N]=\left(k^{*}\left(i^{*}(z)\right) \cup\left(\tilde{\mu}^{\prime} /[N]\right)=\right.$ $k^{*}\left(i^{*}(z)\right)$, as $\tilde{\mu}^{\prime} /[N]=1$ by Theorem 2.2 above. So we have: if $i^{*}(z) \neq 0$ then $\left(k^{*}\left(i^{*}(z)\right) \times 1\right) \cup \tilde{\mu}^{\prime} \neq 0$. Therefore $d_{n}$ is injective on the $(n-1)^{t h}$-row of $E_{n}=E_{2}$.

This yields $E_{n+1}^{i, j}=E_{\infty}^{i, j}=0$ for $j \neq 0$, and $E_{\infty}^{i, 0}=E_{n+1}^{i, 0}=\frac{E_{n}^{i, 0}}{\operatorname{im} d_{n}}=\frac{E_{2}^{i, 0}}{\operatorname{im} d_{n}}=$ $\frac{H^{i}(M \times N ; \mathbb{Z})}{\operatorname{im} d_{n}}$ for all $i$. But, for $0 \leq i \leq n-1$, we have $E_{\infty}^{i, 0}=E_{n+1}^{i, 0}=E_{n}^{i, 0}=E_{2}^{i, 0}=$ $H^{i}(M \times N ; \mathbb{Z})$, and for $n \leq i \leq 2 n, E_{n+1}^{i, 0}=E_{\infty}^{i, 0}=\frac{H^{i}(M \times N ; \mathbb{Z})}{\operatorname{im} d_{n}}$.

Thus

$$
H^{*}(M \times N \backslash \Gamma(f) ; \mathbb{Z}) \cong \frac{H^{*}(M \times N ; \mathbb{Z})}{H^{*}(M \times N ; \mathbb{Z}) \cup \tilde{\mu}^{\prime}} \cong \frac{H^{*}(M \times N ; \mathbb{Z})}{\left(\tilde{\mu}^{\prime}\right)},
$$

as rings, where $\left(\tilde{\mu}^{\prime}\right)$ denotes the ideal of $H^{*}(M \times N ; \mathbb{Z})$ generated by $\tilde{\mu}^{\prime}$. 
If we take cohomology with coefficients in $\mathbb{Q}$, then by an application of the Künneth theorem $H^{*}(M ; \mathbb{Q}) \otimes H^{*}(N ; \mathbb{Q}) \stackrel{\cong}{\rightarrow} H^{*}(M \times N ; \mathbb{Q})$. Hence there is a unique class $\Delta_{f}=\sum_{i=1}^{n}(-1)^{\operatorname{deg}\left(b_{i}\right)} f^{*}\left(b_{i}\right) \otimes b_{i}^{*}$ in $H^{*}(M ; \mathbb{Q}) \otimes H^{*}(N ; \mathbb{Q})$ which maps to $\tilde{\mu}^{\prime}=$ $\sum_{i=1}^{N}(-1)^{\operatorname{deg}\left(b_{i}\right)} f^{*}\left(b_{i}\right) \times b_{i}^{*}=\mu_{f}$ in $H^{*}(M \times N ; \mathbb{Q})$ under this isomorphism, and therefore

$$
H^{*}(M \times N \backslash \Gamma(f) ; \mathbb{Q}) \cong \frac{H^{*}(M \times N ; \mathbb{Q})}{\left(\mu_{f}\right)} \cong \frac{H^{*}(M ; \mathbb{Q}) \otimes H^{*}(N ; \mathbb{Q})}{\left(\Delta_{f}\right)}
$$

as algebras.

A similar argument can be given when $\mathbb{Q}$ is replaced by an arbitrary field.

\section{A CDGA model of $M \times N \backslash \Gamma(f)$}

In this section we prove Theorem 1.4 and Corollary 1.5 by constructing a CDGA model of $\left(1_{M} \times f\right) \circ \Delta_{M}: M \rightarrow M \times M \rightarrow M \times N$ from a CDGA model of $f: M \rightarrow N$. (We refer the reader to [9], [10], [3] and [5] for necessary definitions, notations and results leading to the construction of a CDGA model of the configuration space of two points $F(M, 2))$.

Proof of Theorem 1.4 We first note that a CDGA-model $\left(A_{N}, d_{N}\right) \stackrel{\psi_{f}}{\longrightarrow}\left(A_{M}, d_{M}\right)$ of $f$ determines a CDGA-model $\left(A_{M} \otimes A_{N}, d_{A_{M}} \otimes d_{A_{N}}\right) \stackrel{1_{A_{M}} \otimes \psi_{f}}{\longrightarrow}\left(A_{M} \otimes A_{M}, d_{A_{M}} \otimes\right.$ $d_{A_{M}}$ ) of $1_{M} \times f$, whose verification is left to the reader. This together with the fact that a CDGA-model of $M$ determines a CDGA-model of $\Delta_{M}$ (see, e.g. Example 2.48 on p.73 of [5]) yields the conclusion that a CDGA-model of $f$ determines a CDGA-model $\left(A_{M} \otimes A_{N}\right) \stackrel{1_{A_{M}} \otimes \psi_{f}}{\longrightarrow}\left(A_{M} \otimes A_{M}\right) \stackrel{\nu_{A_{M}}}{\longrightarrow} A_{M}$, defined by $x \otimes y \mapsto$ $\left(v_{A_{M}} \circ\left(1_{A_{M}} \otimes \psi_{f}\right)\right)(x \otimes y)=x . \psi_{f}(y)$, of $\left(1_{M} \times f\right) \circ \Delta_{M}$.

By our hypotheses $H^{1}(M ; \mathbb{Q})=0$ and $H^{1}(N ; \mathbb{Q})=0=H^{2}(N ; \mathbb{Q})$, it follows that for the embedding $\left(1_{M} \times f\right) \circ \Delta_{M}: M \rightarrow M \times N$, which actually embeds $M$ as the graph $\Gamma(f)$ of $f$ in $M \times N$, the hypotheses of Theorem 1.4 of [10], namely $H_{1}\left(\left(1_{M} \times\right.\right.$ $\left.f) \circ \Delta_{M} ; \mathbb{Q}\right)$ is an isomorphism and $H_{2}\left(\left(1_{M} \times f\right) \circ \Delta_{M} ; \mathbb{Q}\right)$ is an epimorphism are satisfied. Therefore by applying Theorem 1.4 of [10] one can determine explicitly a model of $M \times N \backslash \Gamma(f)$ from the above model of $f$, and our result follows.

Proof of Corollary. 1.5 By hypothesis the dimension of $N$ is at least three, so $\operatorname{dim} M=\operatorname{dim} N \geq 3$. Therefore the hypothesis of Corollary 1.5 of [10], namely the codimension of the embedding of $\Gamma(f)$ in $M \times N$ is $\geq 3$, and $H_{*}\left(\left(1_{M} \times f\right) \circ \Delta_{M} ; \mathbb{Q}\right)$ is 2-connected are satisfied. Hence, the rational homotopy type of $M \times N \backslash \Gamma(f)$ is determined by the rational homotopy class of $1_{M} \times f$. But the homotopy class of $1_{M} \times f$ is determined by the homotopy class of $f$; hence the corollary holds. 


\section{The CDGA model of $M \times N \backslash \Gamma(f)$ based on differential-Poincaré-duality-algebra models of $M$ and $N$}

In this section we consider a continuous map $f: M \rightarrow N$ of closed connected oriented manifolds of dimension $n$ with $H^{1}(M ; \mathbb{Q})=0=H^{1}(N ; \mathbb{Q})$ having a CDGA model $\left(A_{N}, d_{A_{N}}\right) \stackrel{\psi_{f}}{\rightarrow}\left(A_{M}, d_{A_{M}}\right)$ in which $\left(A_{M}, d_{A_{M}}\right)$ and $\left(A_{N}, d_{A_{N}}\right)$ are differential connected Poincaré duality algebras of formal dimension $n$ (refer to the para preceding Theorem 1.6 of the introduction).

We note that all the algebras and relevant results of [9], proved by them in the absolute case, can analogously be developed for any given CDGA morphism of Poincaré duality algebras.

Definition 4.1 Let $\left(A_{2}, d_{A_{2}}\right) \stackrel{\psi}{\rightarrow}\left(A_{1}, d_{A_{1}}\right)$ be a morphism of Poincaré duality algebras of formal dimension $n$. Define $\Delta_{\psi}:=(\psi \otimes 1)(\Delta)$, where $\Delta$ is the diagonal class defined in the statement of Theorem 1.6 and the element $\Delta_{\psi}$, which belongs to $\left(A_{1} \otimes A_{2}\right)^{n}$, will be called the class of $\psi$.

As in Proposition 4.3 of [9] the element $\Delta_{\psi}$ does not depend on the choice of the basis $\left\{a_{i}\right\}_{1 \leq i \leq l}$ of $A_{2}$.

Remark 4.2 If $s^{-n} A_{1}$ is the suspension of $A_{1}$ as defined in $\S 2$ of [9] then there is an $\left(A_{1} \otimes A_{2}\right)$-module structure on $s^{-n} A_{1}$ given by

$$
(x \otimes y) \cdot\left(s^{-n} a\right)=(-1)^{n \operatorname{deg}(x)+n \operatorname{deg}(y)+\operatorname{deg}(a) \operatorname{deg}(y)} s^{-n}(x \cdot a \cdot \psi(y))
$$

for homogeneous elements $a, x \in A_{1}$, and $y \in A_{2}$ and an obvious $\left(A_{1} \otimes A_{2}\right)$ module structure on $\left(A_{1} \otimes A_{2}\right)$. In other words the $\left(A_{1} \otimes A_{2}\right)$-module structure on $s^{-n} A_{1}$ is obtained from the obvious structure of $A_{1} \otimes A_{1}$-module on $s^{-n} A_{1}$ by transporting it along the CDGA map $1 \otimes \psi: A_{1} \otimes A_{2} \rightarrow A_{1} \otimes A_{1}$.

In view of the above, statements below follow from the corresponding statements of [9], proved there in the absolute case.

1. Let $\psi:\left(A_{2}, \omega_{A_{2}}\right) \rightarrow\left(A_{1}, \omega_{A_{1}}\right)$ be a CDGA morphism of oriented Poincaré duality algebras of finite dimension and of formal dimension $n$. Then the map $\widehat{\Delta_{\psi}}: s^{-n} A_{1} \rightarrow A_{1} \otimes A_{2}$, defined by $s^{-n} a \mapsto \Delta_{\psi} \cdot(a \otimes 1)$ is a morphism of $\left(A_{1} \otimes A_{2}\right)$-modules.

2. Let $\psi:\left(A_{2}, d_{A_{2}}, \omega_{A_{2}}\right) \rightarrow\left(A_{1}, d_{A_{1}}, \omega_{A_{1}}\right)$ be a morphism of oriented differential Poincaré algebras of formal dimension $n$. Then $\Delta_{\psi}$ is a cocycle.

3. If $\psi:\left(A_{2}, d_{A_{2}}, \omega_{A_{2}}\right) \rightarrow\left(A_{1}, d_{A_{1}}, \omega_{A_{1}}\right)$ is a morphism of connected differential oriented Poincaré duality algebras of formal dimension $n$ and $\widehat{\Delta_{\psi}}: s^{-n} A_{1} \rightarrow$ $A_{1} \otimes A_{2}$ is defined by $s^{-n} a \mapsto \Delta_{\psi} \cdot(a \otimes 1)$, then

(a) $\widehat{\Delta_{\psi}}$ is a morphism of $\left(A_{1} \otimes A_{2}\right)$-dgmodules and hence its mapping cone

$$
C\left(\widehat{\Delta_{\psi}}\right):=A_{1} \otimes A_{2} \oplus_{\widehat{\Delta_{\psi}}} s s^{-n} A_{1}
$$

is an $\left(A_{1} \otimes A_{2}\right)$-dgmodule. 
(b) $C\left(\widehat{\Delta_{\psi}}\right)$ is also a $C D G A$ under the following multiplication rules: If $a, a^{\prime}, x, y \in A_{1}$, and $b, b^{\prime} \in A_{2}$,

$$
\begin{aligned}
(a \otimes b) .\left(a^{\prime} \otimes b^{\prime}\right) & =(-1)^{\operatorname{deg}(b) \operatorname{deg}\left(a^{\prime}\right)}\left(a \cdot a^{\prime} \otimes b \cdot b^{\prime}\right), \\
(a \otimes b) .\left(s s^{-n} x\right) & =(-1)^{(n-1)(\operatorname{deg}(a)+\operatorname{deg}(b))} s s^{-n}(a \cdot \psi(b) \cdot x), \\
\left(s s^{-n} x\right) .(a \otimes b) & =s s^{-n}(x \cdot a \cdot \psi(b)), \\
\left(s s^{-n} x\right) .\left(s s^{-n} y\right) & =0 .
\end{aligned}
$$

Definition 4.3 Let $(A, \omega)$ be a connected oriented Poincaré duality algebra of formal dimension $n$ with orientation class $\omega$ (refer to Definition 4.1 of [9]). Since $A^{n} \cong \mathbb{Q}$, there exists a unique element $\mu \in A^{n}$ such that $\omega(\mu)=1$ which called the fundamental class of $A$.

Our goal in this section is to prove Theorem 1.6. Throughout we consider morphisms $\psi:\left(A_{2}, d_{A_{2}}, \omega_{A_{2}}\right) \rightarrow\left(A_{1}, d_{A_{1}}, \omega_{A_{1}}\right)$ of connected differential oriented Poincaré duality algebras of formal dimension $n$.

Lemma 4.4 The ideal $\left(\Delta_{\psi}\right):=\Delta_{\psi} \cdot\left(A_{1} \otimes A_{2}\right)$ generated by $\Delta_{\psi}$ in $A_{1} \otimes A_{2}$ is a differential ideal and the quotient $\left(A_{1} \otimes A_{2}\right) /\left(\Delta_{\psi}\right)$ is a CDGA.

Proof By Remark $4.2(2), d_{A_{1} \otimes A_{2}}\left(\Delta_{\psi}\right)=0$; hence the ideal $\left(\Delta_{\psi}\right)$ is a differential ideal. This implies immediately that the quotient $\left(A_{1} \otimes A_{2}\right) /\left(\Delta_{\psi}\right)$ inherits a $C D G A$ structure.

Lemma 4.5 The map $\widehat{\Delta_{\psi}}$ induces an isomorphism

$$
\widehat{\Delta_{\psi}}: s^{-n} A_{1} \rightarrow\left(\Delta_{\psi}\right) \text {. }
$$

Proof We first show that im $\left(\widehat{\Delta_{\psi}}\right)=\left(\Delta_{\psi}\right)$. Clearly $\operatorname{im}\left(\widehat{\Delta_{\psi}}\right) \subseteq\left(\Delta_{\psi}\right)$. For the reverse inclusion we have

$$
\begin{aligned}
\left(\Delta_{\psi}\right)= & \left\{\Delta_{\psi} \cdot(x \otimes y) \mid x \in A_{1}, y \in A_{2}\right\} \\
= & \left\{\Delta_{\psi} \cdot(-1)^{\operatorname{deg}(x) \operatorname{deg}(y)}(1 \otimes y) .(x \otimes 1) \mid x \in A_{1}, y \in A_{2}\right\} \\
= & \left\{\widehat{\Delta_{\psi}}\left((-1)^{\operatorname{deg}(x) \operatorname{deg}(y)} s^{-n}(\psi(y) . x)\right) \mid x \in A_{1}, y \in A_{2}\right\} \\
& \subseteq \operatorname{im}\left(\widehat{\Delta_{\psi}}\right) .
\end{aligned}
$$

Next we show that $\widehat{\Delta_{\psi}}$ is injective. Let $\omega_{A_{1}}$ and $\omega_{A_{2}}$ be the orientation classes of the Poincaré duality algebras $A_{1}$ and $A_{2}$ respectively. So there exist unique elements $\mu_{A_{1}} \in A_{1}^{n}$ and $\mu_{A_{2}} \in A_{2}^{n}$, fundamental classes of $A_{1}$ and $A_{2}$, such that $\omega_{A_{1}}\left(\mu_{A_{1}}\right)=1$ and $\omega_{A_{2}}\left(\mu_{A_{2}}\right)=1$. Fix a basis $\left\{a_{i}\right\}_{i=1}^{l}$ of $A_{2}$ and its Poincaré dual basis $\left\{a_{i}^{*}\right\}_{i=1}^{l}$. Since $A_{2}^{0}=\mathbb{Q}$ we can assume that $1=a_{1} \in A_{2}^{0}$ and remaining $a_{i}$,'s are of degree $>0$ so that $a_{i} \mu_{A_{2}} \in A_{2}^{>n}=0$ for $i>1$. Also $\omega_{A_{2}}\left(a_{1} \cdot a_{1}^{*}\right)=1$ implies that $1 . a_{i}^{*}=a_{1} \cdot a_{1}^{*}=\mu_{A_{2}}$ so that $a_{1}^{*}=a_{1}^{-1} \cdot \mu_{A_{2}}=\mu_{A_{2}}$. This yields from the definition of $\Delta_{\psi}$ that 


$$
\begin{aligned}
& \Delta_{\psi} \cdot\left(\mu_{A_{1}} \otimes 1\right)=\sum_{i=1}^{l}(-1)^{\operatorname{deg}\left(a_{i}\right)}\left(\psi\left(a_{i}\right) \otimes a_{i}^{*}\right) \cdot\left(\mu_{A_{1}} \otimes 1\right) \\
& =\sum_{i=1}^{l}(-1)^{\operatorname{deg}\left(a_{i}^{*}\right)}(-1)^{n \operatorname{deg}\left(a_{i}^{*}\right)}\left(\psi\left(a_{i}\right) \cdot \mu_{A_{1}} \otimes a_{i}^{*}\right)=\psi\left(a_{1}\right) \cdot \mu_{A_{1}} \otimes a_{1}^{*} \\
& =\psi\left(a_{1}\right) \cdot \mu_{A_{1}} \otimes a_{1}^{-1} \cdot \mu_{A_{2}}=\mu_{A_{1}} \otimes \mu_{A_{2}} \neq 0 .
\end{aligned}
$$

Let $a$ be a non-zero element of $A_{1}^{i}$. By Poincaré duality there exists an element $b \in$ $A_{1}^{n-i}$ such that $a . b=\mu_{A_{1}}$. We have

$$
\begin{aligned}
\widehat{\Delta_{\psi}}\left(s^{-n} a\right) .(b \otimes 1) & =\Delta_{\psi} \cdot(a \otimes 1) \cdot(b \otimes 1)=\Delta_{\psi} \cdot(a \cdot b \otimes 1)=\Delta_{\psi} \cdot\left(\mu_{A_{1}} \otimes 1\right) \\
& =\mu_{A_{1}} \otimes \mu_{A_{2}} \neq 0 .
\end{aligned}
$$

Consider the projection $A_{1} \otimes A_{2} \stackrel{\pi}{\rightarrow}\left(A_{1} \otimes A_{2}\right) /\left(\Delta_{\psi}\right)$. We extend $\pi$ to a map $\hat{\pi}$ : $A_{1} \otimes A_{2} \oplus_{\widehat{\Delta_{\psi}}} s s^{-n} A_{1} \rightarrow\left(A_{1} \otimes A_{2}\right) /\left(\Delta_{\psi}\right)$ by setting $\hat{\pi}\left(s s^{-n} A_{1}\right)=0$.

Lemma 4.6 The map $\hat{\pi}: A_{1} \otimes A_{2} \oplus_{\widehat{\Delta_{\psi}}} s s^{-n} A_{1} \rightarrow\left(A_{1} \otimes A_{2}\right) /\left(\Delta_{\psi}\right)$ defined above is a CDGA quasi-isomorphism.

Proof The map $\hat{\pi}$ can be seen to be a $C D G A$ morphism by a straightforward computation. Since $\widehat{\Delta_{\psi}}$ is injective, we have a short exact sequence

$$
0 \rightarrow s^{-n} A_{1} \stackrel{\widehat{\Delta_{\psi}}}{\longrightarrow} A_{1} \otimes A_{2} \stackrel{\pi}{\rightarrow}\left(A_{1} \otimes A_{2}\right) / \mathrm{im}\left(\widehat{\Delta_{\psi}}\right) \rightarrow 0
$$

Comparing the long cohomology exact sequence corresponding to this exact sequence and that of the mapping cone $s^{-n} A_{1} \stackrel{\widehat{\Delta_{\psi}}}{\longrightarrow} A_{1} \otimes A_{2} \hookrightarrow C\left(\widehat{\Delta_{\psi}}\right):=A_{1} \otimes A_{2} \oplus \widehat{\Delta_{\psi}}$ $s s^{-n} A_{1}$ we get the following commutative diagram:

$$
\begin{aligned}
& \rightarrow H^{q}\left(s^{-n} A_{1}\right) \rightarrow H^{q}\left(A_{1} \otimes A_{2}\right) \rightarrow H^{q}\left(C\left(\widehat{\Delta_{\psi}}\right)\right) \rightarrow H^{q+1}\left(s^{-n} A_{1}\right) \rightarrow H^{q+1}\left(A_{1} \otimes A_{2}\right) \rightarrow \\
& \begin{array}{c}
\downarrow \\
\downarrow H^{q}\left(s^{-n} A_{1}\right) \rightarrow H^{q}\left(A_{1} \otimes A_{2}\right) \rightarrow H^{q}\left(\frac{A_{1} \otimes A_{2}}{\operatorname{im}\left(\Delta_{\psi}\right)}\right) \rightarrow H^{q+1}\left(s^{-n} A_{1}\right) \rightarrow H^{q+1}\left(A_{1} \otimes A_{2}\right) \rightarrow
\end{array}
\end{aligned}
$$

Applying five lemma and using Lemma 4.5 we get that the map

$$
A_{1} \otimes A_{2} \oplus_{\widehat{\Delta_{\psi}}} s s^{-n} A_{1} \stackrel{\hat{\pi}=\pi \oplus 0}{\longrightarrow}\left(A_{1} \otimes A_{2}\right) / \mathrm{im}\left(\widehat{\Delta_{\psi}}\right)=\left(A_{1} \otimes A_{2}\right) /\left(\Delta_{\psi}\right)
$$

is a quasi isomorphism.

We are now ready to prove Theorem 1.6. 
Proof of Theorem. 1.6 We have proved in Lemma 4.4 that $\left(\Delta_{\psi_{f}}\right)$ is a differential ideal. Since $\left(A_{M}, d_{A_{M}}\right)$ and $\left(A_{N}, d_{A_{N}}\right)$ are connected differential Poincaré duality algebras of formal dimension $n$ and since $H^{n}\left(A_{M}, d_{A_{M}}\right)=H^{n}(M ; \mathbb{Q}) \neq 0$ and $H^{n}\left(A_{N}, d_{A_{N}}\right)=H^{n}(N ; \mathbb{Q}) \neq 0$, Proposition 4.8 of [9] implies that $\left(A_{M}, d_{A_{M}}, \omega_{A_{M}}\right)$ and $\left(A_{N}, d_{A_{N}}, \omega_{A_{N}}\right)$ are oriented differential Poincaré duality algebras in the sense of Definition 4.6 of [9] for orientations $\omega_{A_{M}} \in \# A_{M}^{n}$ and $\omega_{A_{N}} \in \# A_{N}^{n}$.

We have proved in $\S 3$ that a CDGA model $\psi_{f}:\left(A_{N}, d_{A_{N}}\right) \rightarrow\left(A_{M}, d_{A_{M}}\right)$ of $f$ gives a CDGA model

$$
\Phi:=v_{A_{M}} \circ\left(1_{A_{M}} \otimes \psi_{f}\right):\left(A_{M} \otimes A_{N}, d_{A_{N}} \otimes d_{A_{M}}\right) \rightarrow\left(A_{M}, d_{A_{M}}\right)
$$

of $\left(1_{M} \times f\right) \circ \Delta: M \rightarrow M \times N$, and is given by $x \otimes y \mapsto x . \psi_{f}(y)$. Set $n=2 m$. The morphism $\Phi$ induces an obvious $\left(A_{M} \otimes A_{N}\right)$-dgmodule structure on $A_{M}$, hence on $s^{-n} \# A_{M}$. By Proposition 4.7 of [9] $s^{-n} \# A_{M}=s^{-m}\left(s^{-m} \# A_{M}\right)$ is isomorphic to $s^{-m} A_{M}$ as an $A_{M}$-dgmodule, therefore also an isomorphism as an $\left(A_{M} \otimes A_{N}\right)$ dgmodule.

By Remark 4.2a the map $\widehat{\Delta_{\psi}}: s^{-n} A_{M} \rightarrow A_{M} \otimes A_{N}$ is a map of $\left(A_{M} \otimes A_{N}\right)$ dgmodules. Moreover, it induces an isomorphism in the cohomology in the top degree, because $\widehat{\Delta_{\psi}}\left(s^{-n} \mu\right)=\mu_{A_{M}} \otimes \mu_{A_{N}}$. Thus $\widehat{\Delta_{\psi}}$ is a shriek map (or, a top-degree map) in the sense of Definition 5.1 of [10].

Let $I_{0}$ be a complement of the cocycles in $\left(A_{M} \otimes A_{N}\right)^{n-3}$ and set $I=I_{0} \oplus$ $\left(A_{M} \otimes A_{N}\right)^{>n-3}$. Let $K_{0}$ be a complement of the cocycles in $\left(s^{-n} A_{M}\right)^{n-2}$ and set $K=K_{0} \oplus\left(s^{-n} A_{M}\right)^{>n-2}$. Consider the quotient

$$
\frac{A_{M} \otimes A_{N} \oplus_{\widehat{\Delta_{\psi}}} s s^{-n} A_{M}}{I \oplus s K} .
$$

By the hypothesis of the theorem concerning $f$, it follows that the embedding $\left(1_{M} \times f\right) \circ \Delta_{M}: M \hookrightarrow M \times N$, which embeds $M$ as $\Gamma(f)$ in $M \times N$, satisfies the hypothesis of Theorem 1.4 of [10], namely that $H_{1}\left(\left(1_{M} \times f\right) \circ \Delta_{M} ; \mathbb{Q}\right)$ is an isomorphism and $H_{2}\left(\left(1_{M} \times f\right) \circ \Delta_{M} ; \mathbb{Q}\right)$ is an epimorphism, hence by Theorem 1.4 of [10] we get that

$$
\left(\frac{A_{M} \otimes A_{N} \oplus_{\widehat{\Delta_{\psi}}} s s^{-n} A_{M}}{I \oplus s K}, \bar{d}\right)
$$

is a CDGA model of $M \times N \backslash \Gamma(f)$.

Comparing the semi-trivial CDGA structure as defined in Definition 4.1 of [10] on $\left(A_{M} \otimes A_{N} \oplus_{\widehat{\Delta_{\psi}}} s s^{-n} A_{M}\right) /(I \oplus s K)$ with the multiplication in the mapping cone given in this section, we conclude that the projection

$$
\left(A_{M} \otimes A_{N} \oplus_{\widehat{\Delta_{\psi}}} s s^{-n} A_{M}, d\right) \rightarrow\left(\frac{A_{M} \otimes A_{N} \oplus_{\widehat{\Delta_{\psi}}} s s^{-n} A_{M}}{I \oplus s K}, \bar{d}\right)
$$

is a CDGA map. Moreover it is a quasi-isomorphism by Lemma 8.6 of [10]. 
Therefore $\left(A_{M} \otimes A_{N} \oplus_{\Delta_{\psi}} s s^{-n} A_{M}, d\right)$ is a CDGA model of $M \times N \backslash \Gamma(f)$. Hence by Lemma 4.6 $\left(\left(A_{M} \otimes A_{N}\right) /\left(\Delta_{\psi}\right), \overline{d_{A_{M}} \otimes d_{A_{N}}}\right)$ is a CDGA model of $M \times N \backslash \Gamma(f)$.

We finally prove Corollary 1.7 on formality of the complement of the graph of a map.

Proof of corollary. 1.7 Since $f: M \rightarrow N$ is formal, $\left(H^{*}(N ; \mathbb{Q}), 0\right) \stackrel{f^{*}}{\rightarrow} .\left(H^{*}(M ; \mathbb{Q}), 0\right)$ is a model of $f$.

Moreover, since $\left(H^{*}(N ; \mathbb{Q}), 0\right)$ and $\left(H^{*}(M ; \mathbb{Q}), 0\right)$ are oriented differential Poincaré duality algebras, by Theorems 1.6 and 1.8 ,

$$
\left(\frac{H^{*}(M ; \mathbb{Q}) \otimes H^{*}(N ; \mathbb{Q})}{\left(\Delta_{f}\right)}, 0\right) \cong\left(H^{*}(M \times N \backslash \Gamma(f) ; \mathbb{Q}), 0\right) \text { (as CDGAs) }
$$

is a CDGA-model of $M \times N \backslash \Gamma(f)$, and hence it is a formal space.

\section{Examples and applications}

In the last section we saw that if $f: M \rightarrow N$ is a formal map of closed connected oriented formal manifolds of dimension $n$ such that $H^{1}(M ; \mathbb{Q})=0=H^{1}(N ; \mathbb{Q})=$ $H^{2}(N ; \mathbb{Q})$, then $M \times N \backslash \Gamma(f)$ is formal (Corollary 1.7). In Proposition 6.6 of [9] Lambrechts and Stanley proved that if $M$ is a closed connected orientable manifold of dimension $n$ such that $M \times M \backslash \Gamma\left(1_{M}\right)=F(M, 2)$ is formal then $M$ is formal. We ask a similar question (this is the converse of Corollary 1.7):

Question 5.1 Given closed connected oriented formal manifolds $M$ and $N$ of dimension $n$ and a continuous map $f: M \rightarrow N$ such that $M \times N \backslash \Gamma(f)$ is formal, is $f$ necessarily a formal map?

The answer is in the negative. Here we construct such an example.

Example 5.2 Consider $S^{7} \stackrel{f=(h, c)}{\longrightarrow} S^{4} \times S^{3}$, where $h: S^{7} \rightarrow S^{4}$ is the Hopf map and $c: S^{7} \rightarrow S^{3}$ is a constant map. It follows from Proposition 2.5 of [1] that any formal map $S^{7} \rightarrow S^{4} \times S^{3}$ must be nullhomotopic; but $f$ is not nullhomotopic. Therefore $f$ is not formal.

Now we show that $S^{7} \times\left(S^{4} \times S^{3}\right) \backslash \Gamma(f)$ is formal. Since $S^{3} \stackrel{j}{\rightarrow} S^{7} \stackrel{h}{\rightarrow} S^{4}$ is a Hopf fibration we have the following commutative diagram [see example (2.68) page 82 of [5] or Chapter 15 of [3]

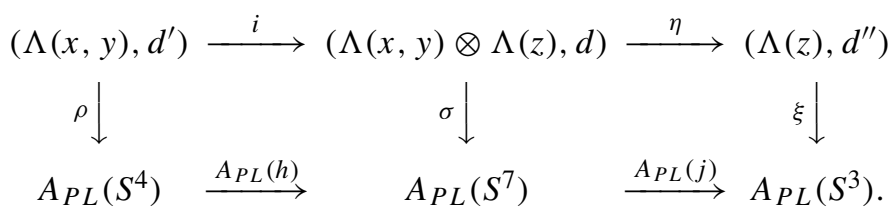

Here $\operatorname{deg}(x)=4, \operatorname{deg}(y)=7$, and $\operatorname{deg}(z)=3 ; d^{\prime} x=0, d^{\prime} y=x^{2}, d x=0$, $d y=x^{2}, d z=x$; and $d^{\prime \prime} z=0$; the morphism $\rho:\left(\Lambda(x, y), d^{\prime}\right) \rightarrow A_{P L}\left(S^{4}\right)$ is the 
minimal model of $S^{4}, \sigma:(\Lambda(x, y) \otimes \Lambda(z), d) \rightarrow A_{P L}\left(S^{7}\right)$ is a quasi-isomorphism, $\xi$ : $\left(\Lambda(z), d^{\prime \prime}\right) \rightarrow A_{P L}\left(S^{3}\right)$ is the minimal model of $S^{3}, i:\left(\Lambda(x, y), d^{\prime}\right) \rightarrow(\Lambda(x, y) \otimes$ $\Lambda(z), d)$ is a relative minimal CDGA and $\eta:(\Lambda(x, y) \otimes \Lambda(z), d) \rightarrow\left(\Lambda(z), d^{\prime \prime}\right)$ is the projection.

From the above diagram we get the following commutative diagram

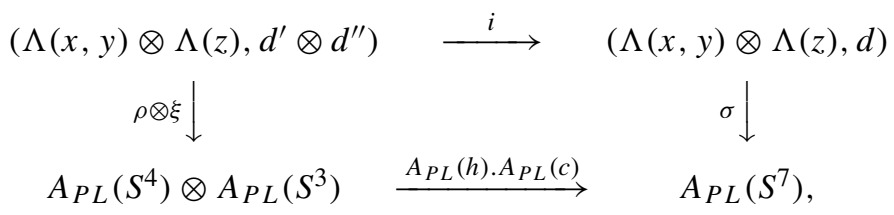

where $i(x)=x, i(y)=y$ and $i(z)=0$.

Let us define CDGAs

$$
(B, 0):=\left(\Lambda\left(e_{4}, e_{3}\right) / I_{B}, 0\right)
$$

where $\operatorname{deg}\left(e_{4}\right)=4 ; \operatorname{deg}\left(e_{3}\right)=3, I_{B}$ is the ideal generated by $e_{4}^{2}$, and

$$
\left(A, d_{A}\right):=\left(\Lambda\left(u_{4}, u_{3}\right) / I_{A}, d_{A}\right)
$$

where $\operatorname{deg}\left(u_{4}\right)=4 ; \operatorname{deg}\left(u_{3}\right)=3, I_{A}$ is the ideal generated by $u_{4}^{2}, d_{A} \overline{u_{3}}=\overline{u_{4}}$, and $d_{A} \overline{u_{4}}=0$. It is easily checked that $(B, 0)$, and $\left(A, d_{A}\right)$ are oriented differential Poincaré duality algebras.

We define morphisms

$$
\rho_{B}:\left(\Lambda(x, y) \otimes \Lambda(z), d^{\prime} \otimes d^{\prime \prime}\right) \rightarrow(B, 0),
$$

where $\rho_{B}(x)=\overline{e_{4}}, \rho_{B}(y)=0, \rho_{B}(z)=\overline{e_{3}}$ and

$$
\rho_{A}:(\Lambda(x, y) \otimes \Lambda(z), d) \rightarrow\left(A, d_{A}\right),
$$

where $\rho_{A}(x)=\overline{u_{4}}, \rho_{A}(y)=0, \rho_{A}(z)=\overline{u_{3}}$.

Clearly $\rho_{B}$ and $\rho_{A}$ are quasi-isomorphisms.

Define $\psi_{f}:(B, 0) \rightarrow\left(A, d_{A}\right)$ by $\psi_{f}\left(\overline{e_{4}}\right)=\overline{u_{4}}, \psi_{f}\left(\overline{e_{3}}\right)=0$ so that the following diagram is commutative.

$$
\begin{array}{cc}
(B, 0)=\left(\Lambda\left(e_{4}, e_{3}\right) / I_{B}, 0\right) \stackrel{\psi_{f}}{\longrightarrow} & \left(\Lambda\left(u_{4}, u_{3}\right) / I_{A}, d_{A}\right)=\left(A, d_{A}\right) \\
\rho_{B} \uparrow & \rho_{A} \uparrow \\
\left(\Lambda(x, y) \otimes \Lambda(z), d^{\prime} \otimes d^{\prime \prime}\right) \stackrel{i}{\longrightarrow} & (\Lambda(x, y) \otimes \Lambda(z), d) .
\end{array}
$$


Using the fact that if $X \stackrel{(k, l)}{\longrightarrow} Y \times Z$ is a continuous map then $A_{P L}(k) \cdot A_{P L}(l)$ : $A_{P L}(Y) \otimes A_{P L}(Z) \rightarrow A_{P L}(X)$ is its model, we get the following commutative diagram

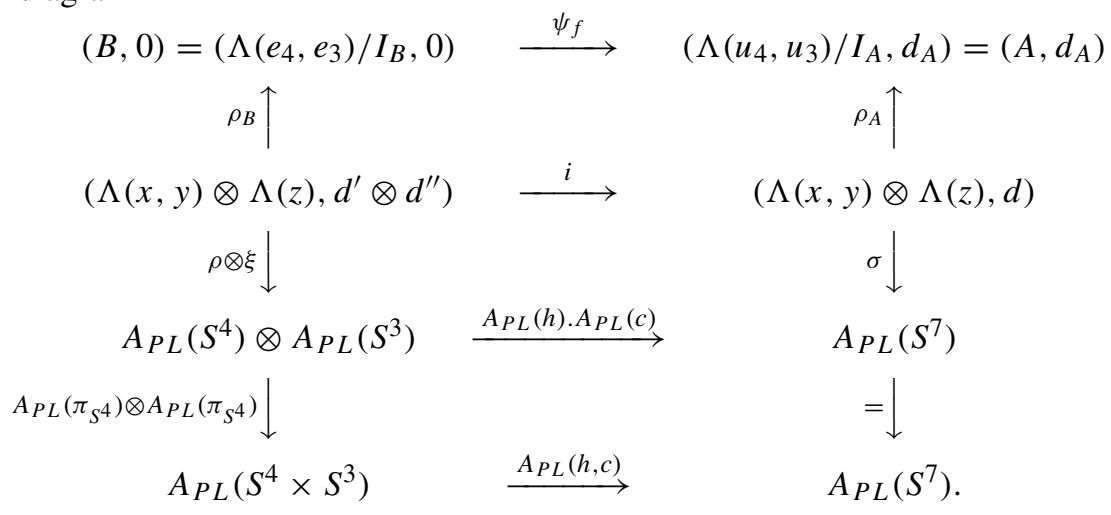

Therefore $\psi_{f}$ is a model of $f=(h, c)$. So, by Theorem 1.6

$$
\left(\frac{A \otimes B}{\left(\triangle_{\psi_{f}}\right)}, \overline{d_{A} \otimes 0}\right)
$$

is a CGDA model of $S^{7} \times\left(S^{4} \times S^{3}\right) \backslash \Gamma(f)$.

Note that $\left\{a_{1}=1, a_{2}=\overline{e_{3}}, a_{3}=\overline{e_{4}}, a_{4}=\overline{e_{4}} \cdot \overline{e_{3}}\right\}$ is a homogeneous basis of $(B, 0)$ with Poincaré dual basis $\left\{a_{1}^{*}=\overline{e_{4}} \cdot \overline{e_{3}}, a_{2}^{*}=\overline{e_{4}}, a_{3}^{*}=\overline{e_{3}}, a_{4}^{*}=1\right\}$, therefore by definition

$$
\begin{aligned}
\triangle_{\psi_{f}} & =\psi_{f}(1) \otimes \overline{e_{4}} \cdot \overline{e_{3}}-\psi_{f}\left(\overline{e_{3}}\right) \otimes \overline{e_{4}}+\psi_{f}\left(\overline{e_{4}}\right) \otimes \overline{e_{3}}-\psi_{f}\left(\overline{e_{4}} \cdot \overline{e_{3}}\right) \otimes 1 \\
& =1 \otimes \overline{e_{4}} \cdot \overline{e_{3}}-\overline{u_{4}} \otimes \overline{e_{3}} .
\end{aligned}
$$

Therefore as a CDGA

$$
\begin{gathered}
\left(\frac{A \otimes B}{\left(\triangle_{\psi_{f}}\right)}, \overline{d_{A} \otimes 0}=\bar{d}\right)=\mathbb{Q}(\overline{1 \otimes 1}) \stackrel{0}{\rightarrow} 0 \stackrel{0}{\rightarrow} 0 \stackrel{0}{\rightarrow} \mathbb{Q}\left(\overline{1 \otimes \overline{e_{3}}}, \overline{\overline{u_{3}} \otimes 1}\right) \\
\stackrel{\bar{d}}{\rightarrow} \mathbb{Q}\left(\overline{1 \otimes \overline{e_{4}}}, \overline{\overline{u_{4}} \otimes 1}\right) \stackrel{0}{\rightarrow} 0 \stackrel{0}{\rightarrow} \mathbb{Q}\left(\overline{\overline{u_{3}} \otimes \overline{e_{3}}}\right) \stackrel{\bar{d}}{\rightarrow} \mathbb{Q}\left(\overline{\overline{u_{3}} \otimes \overline{e_{4}}}, \overline{\overline{u_{4}} \otimes \overline{e_{3}}}, \overline{\overline{u_{4}} \cdot \overline{u_{3}} \otimes 1}\right) \\
\stackrel{\bar{d}}{\rightarrow} \mathbb{Q}\left(\overline{\overline{u_{4}} \otimes \overline{e_{4}}}\right) \stackrel{0}{\rightarrow} 0 \stackrel{0}{\rightarrow} \mathbb{Q}\left(\overline{\overline{u_{4}}} \cdot \overline{\overline{u_{3}} \otimes \overline{e_{3}}}\right) \stackrel{0}{\rightarrow} \mathbb{Q}\left(\overline{\overline{u_{4}}} \cdot \overline{\overline{u_{3}}} \otimes \overline{e_{4}}\right) \stackrel{0}{\rightarrow} 0 \ldots
\end{gathered}
$$

Let $c_{3} \in H^{3}\left(S^{3} ; \mathbb{Q}\right), c_{4} \in H^{4}\left(S^{4} ; \mathbb{Q}\right)$ and $c_{7} \in H^{7}\left(S^{7} ; \mathbb{Q}\right)$ be bases of the respective vector spaces. Then $\left\{b_{1}=1 \otimes 1, b_{2}=1 \otimes c_{3}, b_{3}=c_{4} \otimes 1, b_{4}=c_{4} \otimes c_{3}\right\}$ form a homogeneous basis of $H^{*}\left(S^{4} \times S^{3} ; \mathbb{Q}\right)=H^{*}\left(S^{4} ; \mathbb{Q}\right) \otimes H^{*}\left(S^{3} ; \mathbb{Q}\right)$ with Poincaré dual basis $\left\{b_{1}^{*}=c_{4} \otimes c_{3}, b_{2}^{*}=c_{4} \otimes 1, b_{3}^{*}=1 \otimes c_{3}, b_{4}^{*}=1 \otimes 1\right\}$. We know by 
Theorem 1.8 that

$$
H^{*}\left(S^{7} \times\left(S^{4} \times S^{3}\right) \backslash \Gamma(f) ; \mathbb{Q}\right)=\frac{H^{*}\left(S^{7} ; \mathbb{Q}\right) \otimes H^{*}\left(S^{4} \times S^{3} ; \mathbb{Q}\right)}{\left(\triangle_{f}\right)},
$$

where

$$
\begin{aligned}
\triangle_{f}= & f^{*}(1 \otimes 1) \otimes c_{4} \otimes c_{3}-f^{*}\left(1 \otimes c_{3}\right) \otimes c_{4} \otimes 1+f^{*}\left(c_{4} \otimes 1\right) \otimes 1 \otimes c_{3} \\
& -f^{*}\left(c_{4} \otimes c_{3}\right) \otimes 1 \otimes 1 \\
= & 1 \otimes c_{4} \otimes c_{3} .
\end{aligned}
$$

Therefore as a CDGA

$$
\begin{aligned}
& \left(H^{*}\left(S^{7} \times\left(S^{4} \times S^{3}\right) \backslash \Gamma(f) ; \mathbb{Q}\right), 0\right) \\
& =\mathbb{Q}(\overline{1 \otimes 1 \otimes 1}) \stackrel{0}{\rightarrow} 0 \stackrel{0}{\rightarrow} 0 \stackrel{0}{\rightarrow} \\
& \mathbb{Q}\left(\overline{1 \otimes 1 \otimes c_{3}}\right) \\
& \quad \stackrel{0}{\rightarrow} \mathbb{Q}\left(\overline{1 \otimes c_{4} \otimes 1}\right) \stackrel{0}{\rightarrow} 0 \stackrel{0}{\rightarrow} 0 \stackrel{0}{\rightarrow} \mathbb{Q}\left(\overline{c_{7} \otimes 1 \otimes 1}\right) \stackrel{0}{\rightarrow} 0 \stackrel{0}{\rightarrow} 0 \\
& \quad \stackrel{0}{\rightarrow} \mathbb{Q}\left(\overline{c_{7} \otimes 1 \otimes c_{3}}\right) \stackrel{0}{\rightarrow} \mathbb{Q}\left(\overline{c_{7} \otimes c_{4} \otimes 1}\right) \stackrel{0}{\rightarrow} 0 \ldots
\end{aligned}
$$

Now we define a CDGA morphism

$$
\eta:\left(H^{*}\left(S^{7} \times\left(S^{4} \times S^{3}\right) \backslash \Gamma(f) ; \mathbb{Q}\right), 0\right) \rightarrow\left(\frac{A \otimes B}{\left(\triangle \psi_{f}\right)}, d_{A} \otimes 0\right),
$$

by defining it on generators as follows:

$$
\begin{aligned}
\eta(\overline{1 \otimes 1 \otimes 1}) & =\overline{1 \otimes 1}, \eta\left(\overline{1 \otimes 1 \otimes c_{3}}\right)=\overline{1 \otimes \overline{e_{3}}}, \eta\left(\overline{1 \otimes c_{4} \otimes 1}\right)=\overline{1 \otimes \overline{\overline{e_{4}}}}+\overline{\overline{u_{4}} \otimes 1}, \\
\eta\left(\overline{c_{7} \otimes 1 \otimes 1}\right) & =\overline{\overline{u_{4}} \cdot \overline{u_{3}} \otimes 1}, \eta\left(\overline{c_{7} \otimes 1 \otimes c_{3}}\right)=\overline{\overline{u_{4}}} \cdot \overline{u_{3} \otimes \overline{e_{3}}}, \eta\left(\overline{c_{7} \otimes c_{4} \otimes 1}\right) \\
& =\overline{\overline{u_{4}}} \cdot \overline{u_{3}} \otimes \overline{\bar{u}_{4}}
\end{aligned}
$$

It is checked easily that $\eta$ is a quasi-isomorphism. Therefore $\left(H^{*}\left(S^{7} \times\left(S^{4} \times S^{3}\right) \backslash\right.\right.$ $\Gamma(f) ; \mathbb{Q}), 0)$ is a CDGA model of $S^{7} \times\left(S^{4} \times S^{3}\right) \backslash \Gamma(f)$. Hence $S^{7} \times\left(S^{4} \times S^{3}\right) \backslash \Gamma(f)$ is a formal space.

Remark 5.3 It is known (see e.g. Lemma 6.3 of [9]) that if $M$ is a simply connected closed manifold, if $x \in M$, and if $M \backslash\{x\}$ is formal, then $M$ is formal.

Against this background we may ask the following question.

Question 5.4 Let $f: M \rightarrow N$ be a continuous map between simply connected formal manifolds and let $x \in M$. Suppose that $\left.f\right|_{M \backslash\{x\}}: M \backslash\{x\} \longrightarrow N \backslash\{f(x)\}$ is formal. Is it necessarily true that $f$ is formal?

The following example shows that the answer to this question is in the negative. 
Example 5.5 Take $M=S^{3}, N=S^{2}$ and $f$ the Hopf map $h: S^{3} \rightarrow S^{2}$. Now $S^{3} \backslash\{(0,0,0,1)\} \cong \mathbb{R}^{3}$ and $S^{2} \backslash\{(0,0,1)\} \cong \mathbb{R}^{2}$. Since any continuous map form $\mathbb{R}^{3} \rightarrow \mathbb{R}^{2}$ is formal, $\left.h\right|_{S^{3} \backslash\{(0,0,0,1)\}}: S^{3} \backslash\{(0,0,0,1)\} \longrightarrow S^{2} \backslash\{(0,0,1)\}$ is formal. But $h$ is not formal.

We end the paper by recording a few simple applications of Theorem 1.6 and Corollary 1.7:

Application 5.6 1. Let $M$ and $N$ be two simply connected closed formal manifolds of dimension $n$. For any element $y \in N$, since the constant map $f_{y}: M \rightarrow N$ defined by $f_{y}(x)=y$ for all $x \in X$ is formal, by Corollary 1.7, $M \times N \backslash \Gamma(f)$ is formal. But $M \times N \backslash \Gamma(f)=M \times(N \backslash\{y\})$. Therefore $M \times(N \backslash\{y\})$ is formal.

2. Let $M$ be a simply connected closed formal manifold of dimension $n$. Since the identity map $f=1_{M}: M \rightarrow M$ is formal, by Corollary $1.7, M \times M \backslash \Gamma(f)$ is formal. But $M \times M \backslash \Gamma(f)=F(M, 2)$. Therefore $F(M, 2)$ is formal, which is one of the main results (Corollary 6.1) in [9].

3. Let $M$ and $N$ be two simply connected Lie groups of dimension $n$. It is known that the minimal model of a Lie group is of the form $(\Lambda V, 0)$. Hence Lie groups are formal and continuous maps between Lie groups are also formal. Therefore, by Corollary 1.7, for a continuous map $f: M \rightarrow N, M \times N \backslash \Gamma(f)$ is formal.

Acknowledgments The authors would like to thank the referee for many valuable suggestions. Thanks are also due to Prof. M. B. Rege for suggestions in shortening the presentation.

\section{References}

1. Arkowitz, M.: Formal differential graded algebras and homomorphisms. J. Pure Appl. Algebra 51, 35-52 (1988)

2. Félix, Y.: Mapping spaces between manifolds and the evaluation map. Proc. Am. Math. Soc. 139(10), 3763-3768 (2011)

3. Félix, Y., Halperin, S., Thomas, J.C., Rational homotopy theory, graduate texts in mathematics, 205 Springer (2001)

4. Félix, Y,. Tanré, D.: Formalite d'une application et suite spectrale d'Eilenberg-Moore, Algebraic topology rational homotopy, Lect. Notes in Math., 1318, In: Proceedings, Louvain-la-Neuve, 1986, S.V. pp. 99-123 (1988)

5. Félix, Y., Opera, J., Tanré, D., Algebraic models in geometry, Oxford graduate texts in mathematics, Oxford University Press, p. 17 (2008)

6. Greenberg, M.J., Harper, J.R.: Algebraic topology—a first course, The Benjamin/Cummings Publishing Co. (1981)

7. Kirby, R.C., Siebenmann, L.C.: Foundational essays on topological manifolds. Annals of mathematics studies, smoothings and triangulations, Princeton University Press (1977)

8. Kister, J.M.: Microbundles are fibre bundles. Ann. Math. 80, 190-199 (1964)

9. Lambrechts, P., Stanley, D.: The rational homotopy type of configuration spaces of two points. Ann. Inst. Fourier (Grenoble) 54(4), 1029-1052 (2004)

10. Lambrechts, P., Stanley, D.: Algebraic models of Poincaré embeddings. Algebra. Geom. Topol. 5(9), 135-182 (2005)

11. Lambrechts, P., Stanley, D.: Poincaré duality and commutative differential graded algebras, (2008). arXiv: 0701309v3 [math.AT]

12. Lemaire, J.-M., Sigrist, F.: Sur les invvariants d'homotopie rationnelle liés à la L.S. catégorie. Comment. Math. Helvetici 56, 103-122 (1981)

13. Merle, P.: Formalité des espaces et des applications continues. Thèse, Nice (1983)

14. Milnor, J.W.: Microbundles, Part I, Topology 3. Supplement I, 53-80 (1964) 
15. Milnor, J.W., Stasheff, J.D.: Characteristic classes, annals of mathematics studies. No. 76, Princeton University Press (1974)

16. Papadima, S.: On the formality of maps. Ann. Univ. Timisoara. Seria Math. 20, 30-40 (1982)

17. Shiga, H.: Rational homotopy type and self maps. J. Math. Soc. Japan 31, 427-434 (1979)

18. Shiga, H.: Rational homotopy type of cofibrations. SMF Aster. 113-114, 298-307 (1984)

19. Spanier, E.H.: Algebraic Topology. Tata-McGraw Hill Publ. Company (1977)

20. Thomas, J.C.: Eilenberg-Moore models for fibrations. Trans. Am. Math. Soc. 274(1), 203-225 (1982)

21. Totaro, B.: Configuration spaces of algebraic varieties. Topology 35, 1057-1067 (1996)

22. Vigue-Poirrier, M.: Formalité d'une application continue, C.R.A.S. Paris, t 289 (1979) 